\title{
Improving GIS Disconnectors for Future HVDC Applications
}

\author{
Andreas Ritter, Student Member, IEEE, Ueli Straumann, Senior Member, IEEE, \\ and Christian M. Franck, Senior Member, IEEE
}

\begin{abstract}
The use of standard HVac disconnectors for bustransfer switching in future HVdc gas insulated switchgear (GIS) has been demonstrated successfully in past research through simulation and testing. Prolonged arcing times through reignitions caused by high frequency harmonics as well as the proposed extension of GIS disconnector switching cases, however, create an HVdc-specific demand for more advanced disconnector concepts capable of withstanding higher switching stresses and increased recovery voltages. A spring-loaded disconnector designed for use in HVac mixed technology switchgear (MTS) was found to shorten the time to first current zero through an accelerated increase of the arcing voltage compared to standard disconnectors. Despite its increased switching performance, the reignition characteristics of this fast-acting disconnector were found to largely follow the previously established extinction criterion, which enables further simulation of similar technologies for HVdc applications. To additionally combat the issue of contamination of HVdc GIS by electrode erosion products, a prototype disconnector was improved using permanent magnets to evoke arc rotation. Erosion tests in air have shown an effective reduction of electrode erosion for long arcing times at currents typical for disconnector switching while bus-transfer tests revealed promising enhancements of the reignition behavior.
\end{abstract}

Index Terms-HVdc, disconnector, bus-transfer, currentinterruption, gas insulated substations, arc rotation

\section{INTRODUCTION}

G AS insulated switchgear (GIS) for HVdc and its associated phenomena have been the focus of research and development efforts for more than thirty years, with a special interest in gas and solid insulation as well as their interfaces [1]. Despite the advantages of GIS, such as greatly reduced space requirements and resistance against contamination, only very few installations exist today [2], [3]). One application which could particularly benefit from these advantages is the connection of large scale offshore wind projects to multi-terminal $\mathrm{HVdc}$ (MTdc) grids, due to the high cost of platform space and the benefits of $\mathrm{HVdc}$ submarine cables [4]. After decades of research (reviewed in [5]), the announcements of commercially available $\mathrm{HVdc}$ circuit breakers by manufacturers [6], [7] and their recent integration into one of the first MTdc grids in Zhoushan [8], [9] mark the achievement of one of the key enabling factors of MTdc grids. Compared to circuit breakers, disconnectors

A. Ritter and C. M. Franck are with the Power Systems and High Laboratories (EEH), ETH Zurich, Zurich 8092, Switzerland (e-mail: rittera@ethz.ch).

U. Straumann is with ABB Switzerland Ltd.

This work was financially supported by ABB Switzerland Ltd.

Manuscript received ?; revised?

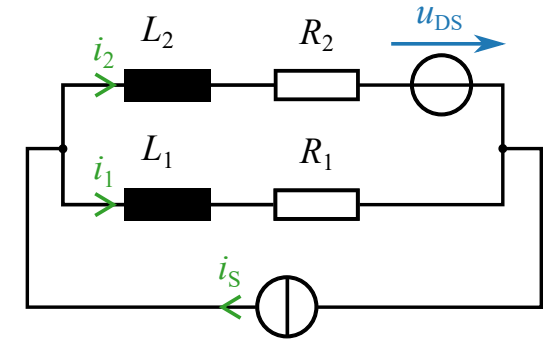

Fig. 1. Simplified equivalent circuit diagram for bus-transfer switching, in which the disconnector is modelled as a current and time dependant voltage source $u_{\mathrm{DS}}$ as used in prior investigations [11], [13], [14].

face lower requirements in terms of switching power but are nevertheless of critical importance for substation and grid operation. Their applications in $\mathrm{HVdc}$ converters have been well documented [10], however, MTdc-specific research of requirements and available technology for use in substations remains rare. The use of disconnectors to transfer load currents between parallel conductors such as bus bars of a substation or submarine cables in an MTdc grid has been investigated by Kanngiesser [11], Vinson [12] and the authors [13]. The findings of long arcing times without current zeroes for large inductances and high harmonic contents leading to multiple reignitions present $\mathrm{dc}$ specific challenges, which have to be investigated further and which can be mitigated by improved disconnector designs.

\section{SCOPE OF RESEARCH}

In HVac GIS, disconnectors have a long history of operation at high reliability rates [15], thus it is sensible to start with established designs and developments when studying requirements and proposing changes for HVdc specific disconnectors. In prior research on the fundamentals of bus-transfer switching in HVac [14] and HVdc GIS [13] employing widely used GIS disconnectors with slow-moving and unblown electrode systems, the authors have identified a correlation between the instantaneous recovery voltage $u_{\mathrm{RV}}$ and the predicted arc voltage $u_{\text {arc }}$ at a current zero at time $t_{0}$, which ensures an extinction of the switching arc:

$$
u_{\mathrm{RV}}\left(t_{0}\right) \leq u_{\mathrm{arc}}\left(t_{0}\right) .
$$

This extinction criterion is based on the finding that in such disconnectors, the conditions for reignition at current zero are almost always assured, so that extinction is only certain if the circuit is unable sustain the arc voltage further. 
For this bus-transfer switching case, the circuit consists of two different lengths $\left(d_{1}, d_{2}\right)$ of substation bus which can be modelled as a parallel connection of two inductances $\left(L_{1}, L_{2}\right)$ and resistances $\left(R_{1}, R_{2}\right)$ as shown in Fig. 1. The switching current $i_{2}$ can be simulated for any source current $i_{\mathrm{S}}$ by numerically solving the resulting differential equation as long as the arc voltage function is known and the bus properties $\left(L^{\prime}\right.$, $\left.R^{\prime}\right)$ as well as the bus lengths are given. While current zeros occur naturally in ac bus-transfer, they have to be generated by the arc voltage in dc cases. The time to first current zero in cases with harmonic-free dc current have been calculated to be on the order of $5 \mathrm{~ms}$ at a pre-switching current of $2 \mathrm{kA}$ for typical configurations of GIS. With increased size $\left(d_{1}, d_{2}\right)$, voltage level $\left(L^{\prime}\right)$ and pre-switching current $\left(i_{\mathrm{S}} \cdot d_{1} /\left(d_{1}+d_{2}\right)\right)$, these times become longer [13].

Another potential application of HVdc disconnectors, which can be modelled by the equivalent circuit diagram of Fig. 1, is that of line transfer under load as simulated by Vinson in [12]. Their simulations found that even a disconnector with substantially faster moving electrodes resulted in arcing times two orders of magnitude longer than for bus-transfer.

The erosion of electrode surfaces by such switching arcs has been studied extensively in the past [16]-[18]. The combination of electrode erosion and mechanical abrasion of moving parts leads to the production of solid particles inside GIS vessels, especially in disconnectors. Resulting effects such as elevated charge transport in the insulating gas and along insulator surfaces [19], lowered dielectric withstand [20], [21], and particle movement [22]-[24] have been well researched, especially for HVac system under dc stress (i.e. trapped charges) and specifically for $\mathrm{HVdc}$ systems. Therefore, reducing the production of particles in disconnectors sustaining repeated operation is an important development goal. In the following, two methods to achieve this goal are evaluated experimentally: A commercially available fast-acting HVac GIS disconnector used in mixed technology switchgear (MTS) and a purposebuilt disconnector testbed with variable opening speed using permanent magnets for magnetic arc driving in atmospheric air.

\section{Test Setup AND Evaluation}

The complete test circuit used to investigate both types of disconnectors is shown in Fig. 2. The source current is provided by the flexible pulsed dc source (FPDCS), which has previously been used for research in HVdc circuit breakers among other applications [25]. FPDCS is composed of three identical buck converter modules $\left(M_{1}, M_{2}, M_{3}\right)$ featuring individually adjustable inductances $\left(L_{1}^{\mathrm{M}}, L_{2}^{\mathrm{M}}, L_{3}^{\mathrm{M}}\right)$ connected in parallel. Each module delivers up to $1 \mathrm{kA}$ of pulsed current at switching frequencies between $0.1 \mathrm{kHz}$ to $10 \mathrm{kHz}$. A detailed review of the working principle, capabilities and applications was recently presented by the authors [26]. The full bustransfer loop consists of two parallel GIS bus equivalents ( $L_{1}, R_{1}$ and $L_{2}, R_{2}$ ), which can be adjusted to represent between $15 \mathrm{~m}$ to $1360 \mathrm{~m}$ of typical $420 \mathrm{kV}$ HVac GIS. The same configuration has been used successfully to study the fundamentals of bus-transfer of slow-moving disconnectors

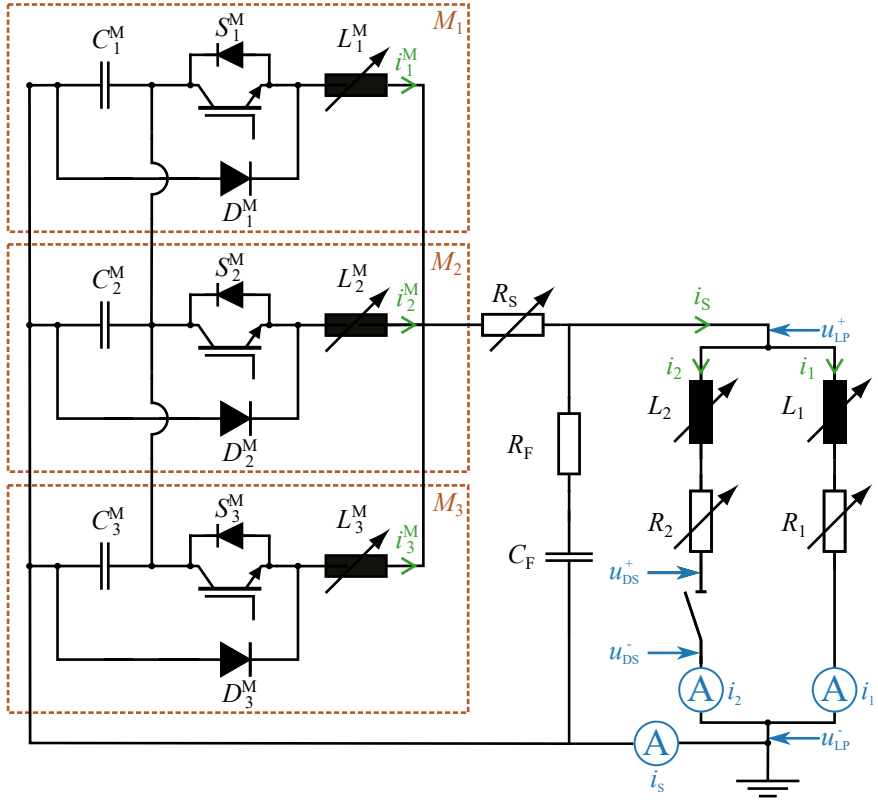

Fig. 2. Equivalent circuit diagram of the flexible pulsed dc source connected to the full bus-transfer loop via an adjustable source resistor and low pass filter including all points of voltage and current measurements (blue).

in ac [14] and dc [13]. For the investigation of disconnectors at higher recovery voltages, a low pass filter $\left(R_{\mathrm{F}}\right.$, $C_{\mathrm{F}}$ ) was implemented, mitigating high frequency oscillations, which are otherwise excited by large changes of the current gradient between the bus-transfer inductances and parasitic capacitances of the FPDCS modules. By blocking frequencies above $500 \mathrm{kHz}$, this filter also creates a predictable low pass characteristic apparent especially during large step changes in the recovery voltage across the open disconnector.

The measurements captured during each test include the absolute voltages on either side of the disconnector $\left(u_{\mathrm{DS}}^{+}\right.$, $\left.u_{\mathrm{DS}}^{-}\right)$and on either side of the entire bus-transfer loop $\left(u_{\mathrm{LP}}^{+}\right.$, $\left.u_{\mathrm{LP}}^{-}\right)$using high-frequency $1: 100$ dividers. The currents $i_{1}$, $i_{2}$ and $i_{\mathrm{S}}$ are measured using current transducers according to the HOKA principle [27] at frequencies between $0 \mathrm{~Hz}$ and $1 \mathrm{MHz}$. Additionally, high speed images at up to $200 \mathrm{kfps}$ were captured during many tests. The evaluation of the measured data follows algorithms established for previous tests of slowmoving disconnectors [13], [14], [28]. Open-circuit (OC) tests, during which the disconnector remains in open position, are used to assess the recovery voltage and to tune the bus-transfer parallel inductance $L_{1}$ as well as the source resistance $R_{\mathrm{S}}$. Short-circuit (SC) tests see the disconnector remain closed in order to tune $R_{1}, L_{2}$ and $R_{2}$ and to find a prediction for the pre-switching current distribution. Using these values, the current distribution between $i_{1}$ and $i_{2}$ as well as the recovery voltage $u_{\mathrm{RV}}$ can be calculated for any test, to aid in the manual evaluation of current zeros. As an additional benefit of the low pass filter, $u_{\mathrm{RV}}$ can be approximated for any point in time of any measurement using $i_{\mathrm{S}}^{\mathrm{LP}}$, a digitally filtered version of the source current $i_{\mathrm{S}}$ in combination with $R_{1}$ and $L_{1}$ calculated from OC measurements, according to the following 
expression.

$$
u_{\mathrm{RV}}(t) \approx R_{1} i_{\mathrm{S}}^{\mathrm{LP}}(t)+L_{1} \frac{d i_{\mathrm{S}}^{\mathrm{LP}}(t)}{d t}
$$

\section{FAST-ACTING Disconnector: SETUP}

In HVac MTS, air insulated buses are linked by gas insulated circuit breakers, disconnectors, and earthing switches, primarily to save space [29]. Disconnectors designed for this application are capable of withstanding up to ten times higher recovery voltages than GIS disconnectors, due to the larger substation dimensions and the increased inductance per unit length. One example of such a disconnector is presented in a 2013 patent [30] to have a spring-loaded opening mechanism inside the pressure vessel in order to create high opening speed. The accelerated contact opening causes a faster increase of the arc voltage compared to traditional, slowmoving disconnectors. In HVdc GIS, a higher arc voltage translates to a lower time to first current zero for a given configuration as well as an increased likelihood of fulfilling the extinction criterion of eq. (1) during bus-transfer, as previously simulated [13]. The disconnector used in this research is an ABB TK3S designed for application in $420 \mathrm{kV}$ MTS substations. It closely follows the aforementioned patent. Due to its intricate electrode separation mechanism, the minimum electrode separation distance has been estimated by means of a FEM tool fed with the measured stroke in dependence of time. First, the electrode deformation during gliding and oscillation after release has been simulated with a structural mechanics module, with electrode pressures being formulated by the penalty method. Second, this mechanical solution has been post-processed, by means of an extrusion-function applied on one electrode side, defining the distance field in the vicinity of this electrode; minimizing it on the contour of the other electrode-side reveals the minimum distance between
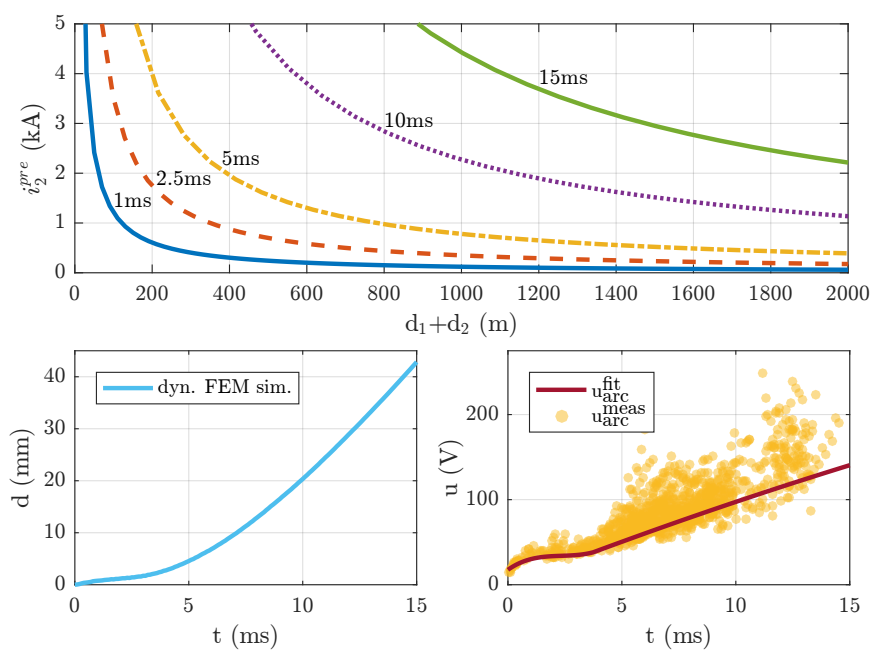

Fig. 3. Top: Time to first current zero for harmonic free dc at varying combinations of pre-switching current equal to $80 \%$ of $i_{\mathrm{S}}$ and total bus lengths $d_{1}+d_{2}$. Bottom, left: Minimum electrode separation estimation based on dynamic FEM simulation. Bottom, right: Arc voltages averaged over a period of $0.5 \mathrm{~ms}$ before every current zero ( $\mathrm{u}_{\mathrm{arc}}^{\text {meas }}$ ) and the two-part fit of data points at currents above $500 \mathrm{~A}\left(\mathrm{u}_{\mathrm{arc}}^{\mathrm{fit}}\right)$. the electrodes for every time-step. Clearly, the arc does not necessarily have to exhibit arc roots resulting simply from the shortest distance between the electrodes. Especially, as there is lateral movement of the electrodes, resulting in arc elongation if the arc roots do not constantly follow the points of shortest distance between the two electrodes. Moreover, longer arcs tend to exhibit instabilities or protuberances, which elongate the arc column independent of the electrode distance, thus increasing the arc voltage further. The minimum electrode separation distance calculated using this method is shown in Fig. 3 (bottom left).

\section{FAST-ACTING DISCONNECTOR: RESULTS}

In order to simplify analysis and simulation of bus-transfer measurements using this disconnector as well as to to verify the dynamic FEM simulations, a two-part polynomial fit was applied to the results of all tests. Arc voltages recorded at currents higher than $500 \mathrm{~A}$ were averaged over $0.1 \mathrm{~ms}$ segments and fit to a fourth order polynomial for the time of lateral movement phase (i.e. $t<3.5 \mathrm{~ms}$ ) and a second order polynomial for the actual electrode separation at times larger than $3.5 \mathrm{~ms}$. In Fig. 3 (bottom right) this fit is compared to all voltages recorded within $0.5 \mathrm{~ms}$ of a current zero, i.e. at low currents. It is evident, that $u_{\text {arc }}^{\text {fit }}$ is a good estimation of the lowest arc voltage as a function of time. In order to estimate time to first current zero, $u_{\text {arc }}^{\text {fit }}$ can be used as a function of voltage of time to simulate $i_{2}$ in the equivalent circuit diagram of Fig. 1. In Fig. 3 (top), the results of this estimation for varying combinations of bus-transfer loop and pre-switching current are presented. It is evident that the fast increase in arc voltage caused by the spring-based contact separation is effective at creating short first current zero times even for high currents and long bus-transfer loops, especially compared to traditional, slow-moving disconnectors. In order to analyze dc bus-transfer performance including reignitions of this particular fast-acting disconnector, more than 400 individual tests were performed using the circuit shown in Fig. 2. Each current zero was manually categorized as extinction of the switching arc or as one of the following three different types of reignitions:

1) Immediate reignition: Switching current crosses zero without any delay. Disconnector voltage is equal to arc voltage immediately before and after current zero. Total number evaluated: 337.

2) Delayed reignition: Switching current remains zero for some delay time before reignition. Disconnector voltage is equal to recovery voltage during delay, after a sudden change in recovery voltage arc voltage appears. Total number evaluated: 58 .

3) Charging reignition: Switching current remains zero for some delay time before reignition. Disconnector voltage is equal to recovery voltage until it switches to arc voltage without a large change. Total number evaluated: 342.

While immediate reignitions were the default reignition type in previous analyses of slow-moving disconnectors in ac and dc conditions at comparably low recovery voltages, they have 
been surpassed in frequency by charging reignitions in tests of the fast-acting disconnector. This new type of reignition is a direct result of the integration of the aforementioned low pass filter, which slows down step-changes in the recovery voltage. During charging reignitions, instead of immediate jumps between arc voltages of different polarity, the voltage across the disconnector changes from arc voltage towards the recovery voltage with an exponential time-constant of approximately $5 \mu \mathrm{s}$. Furthermore, the use of large $L_{1}$ in combination with high source current gradients influences behavior at current zero as well, as it leads to an interaction between the bus-transfer loop inductances $L_{1}$ and $L_{2}$ with
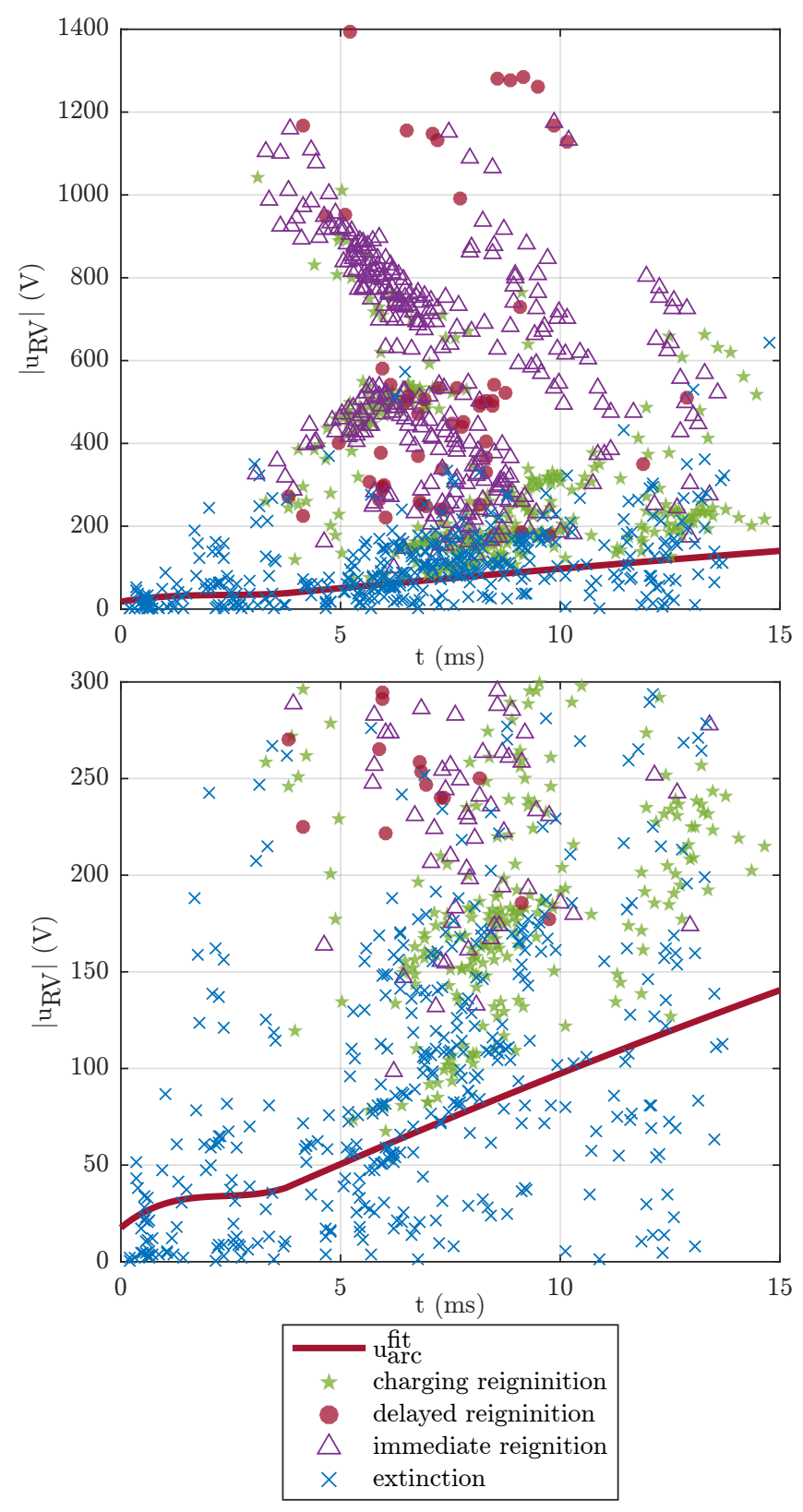

Fig. 4. Absolute recovery voltages of all current zeros recorded during tests of the fast-acting GIS disconnector categorized into different reignition types and extinction. Top: Overview of entire $\left|u_{\mathrm{RV}}\right|$ range. Bottom: Zoom onto voltage range immediately surrounding $u_{\mathrm{arc}}^{\mathrm{fit}}$. the source inductances $L_{1}^{\mathrm{M}}, L_{2}^{\mathrm{M}}$, and $L_{3}^{\mathrm{M}}$. When $i_{2}$ is nonzero, the equivalent inductance of the full bus-transfer loop is approximately equal to $L_{2}$, however, when no current is flowing across the disconnector, the inductance of the bustransfer loop is equal to $L_{1}$. If the difference of these two inductances is in a similar order of magnitude as the smallest source inductance, the change from non-zero to zero current can influence the source current gradient and thus the recovery voltage. The measured recovery voltage $\left(u_{\mathrm{DS}}\right)$ corresponds to zero current across the disconnector (i.e. $L_{1}$ as only load of the current source). However, the recovery voltage relevant for the extinction criterion of eq. (1) is that of a non-zero $i_{2}$ (i.e. $L_{2}$ as load inductance). Therefore, a pre-determined correction factor was applied to extinctions. For charging reignitions and delayed reignitions only the recovery voltages calculated immediately after reignition are used for the same reason.

The results of all evaluated current zeros are presented in Fig. 4. Most of the reignitions occurring at recovery voltages close to the arcing voltage are of charging type, due to the low pass filter's mechanism of slowing down small voltage steps. At recovery voltages more than approximately $200 \mathrm{~V}$ above the predicted arc voltage, immediate reignitions start to dominate. For most cases of delayed reignition, the recovery voltage at current zero is below the predicted arc voltage at that time, thus fulfilling the extinction criterion of eq. (1). However, after a short delay of up to $110 \mu$ s, a sudden change in the recovery voltage to a level otherwise associated with immediate reignitions leads to a delayed reignition. Therefore, the assumption that the conditions for reignition are present only at current zero has to be extended to some time after current zero. The majority of delayed reignitions have been found to occur within $50 \mu \mathrm{s}$ but delays of more than twice that duration have been recorded on rare occasions. For a conclusive characterization of mechanism and likelihood of reignition as a function of delay and voltage for this particular disconnector, more detailed measurements would have to be performed.

These findings are especially important for HVdc applications with high harmonic current contents and thus intermittently high recovery voltages, as well as for applications in which the total arcing time needs to be minimized by a rapidly increasing arc voltage. Fast-acting disconnectors built for use in HVac MTS, therefore, present a suitable starting point for the development of disconnectors addressing these HVdcspecific challenges.

\section{Disconnector Testbed}

Due to the limitations in variability of influencing factors imposed by a production disconnector, requirements for a specialized disconnector testbed were collected. The testbed was aimed at exploring dc-specific improvements to switching performance in an automated fashion with minimal changes to established disconnector mechanics:

1) Large range of opening speeds, adjustable without mechanical changes to electrode system of classic, slowmoving disconnectors, i.e. annular rings.

2) Use of common arcing electrodes of GIS disconnectors in a way that facilitates exchange between tests. 


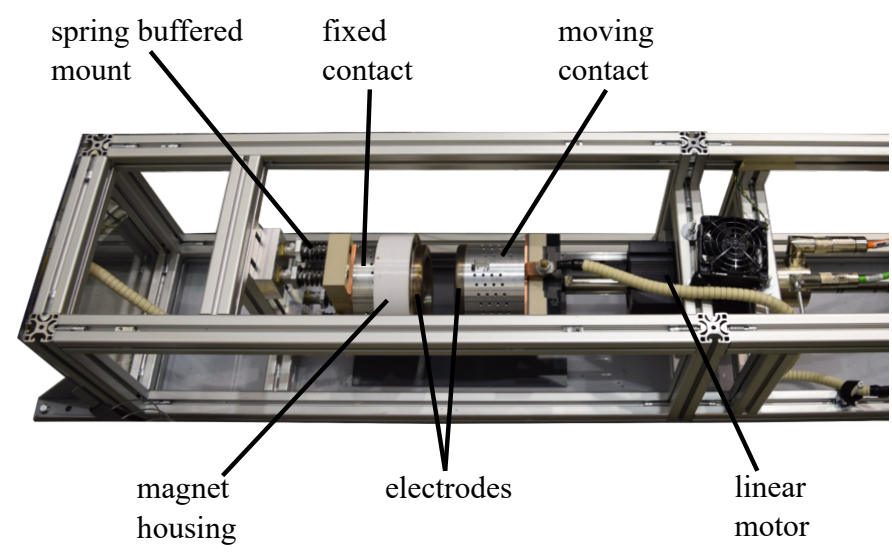

Fig. 5. Top-down view of the disconnector testbed with mounted electrodes and magnets in a single-sided arrangement [31].

3) Application of magnetic arc driving to reduce electrode erosion with minimal changes to contact system.

4) Good observability of switching arc from various angles by high speed camera.

5) Possibility to perform tests in ambient air as well as in pressurized gas vessels.

The realization of these requirements is presented in Fig. 5. The testbed is built using GIS disconnector-specific, annular tungsten-copper $(80 \% \mathrm{~W}, 20 \% \mathrm{Cu})$ arcing electrodes of $94 \mathrm{~mm}$ inner and $128 \mathrm{~mm}$ outer diameter as shown in Fig. 6 . These electrodes are fixed to cylindrical aluminium mounts by four screws each; concentric grooves allow precise orientation. The aluminium mounts are electrically isolated from a spring buffered mount on one side (fixed contact) and motor on the other side (moving contact) by a set of polyether ether ketone (PEEK) plates designed to bend less than $1 \mathrm{~mm}$ at maximum force. The opening speed requirements are fulfilled by a linear motor providing a maximum acceleration force of $1120 \mathrm{~N}$, capable of peak speeds of $4 \mathrm{~m} \mathrm{~s}^{-1}$ when pulling the entire moving contact. To increase the initial speed of electrode separation without the use of abrasion-prone sliding electrodes, a spring buffered mount with an end stop was constructed (cf. left side of Fig. 5). This mount allows the fixed contact to travel with the moving contact for the first few millimeters before electrode separation occurs at up to $1.25 \mathrm{~m} \mathrm{~s}^{-1}$. The entire testbed is built to be small enough to fit inside a typical $550 \mathrm{kV}$ GIS vessel to allow tests in pressurized gases in the future. For the scope of this research, however, tests were performed in ambient air exclusively.

The use of transverse magnetic fields to continuously move an electric arc on the electrode surface has been studied regularly in the past [32], often with the goal of reducing localized heating and electrode erosion [33]. It has also been suggested as a method to improve switching performance of disconnectors [34]. The majority of published concepts rely on one or more coils in the vicinity of the arcing electrodes to create a transverse magnetic field using the switching current. The resulting Lorentz force acting on the arc is thus proportional to the square of the switching current, which can make low-current switching cases such as bustransfer difficult. Additionally, adding a coil powerful enough

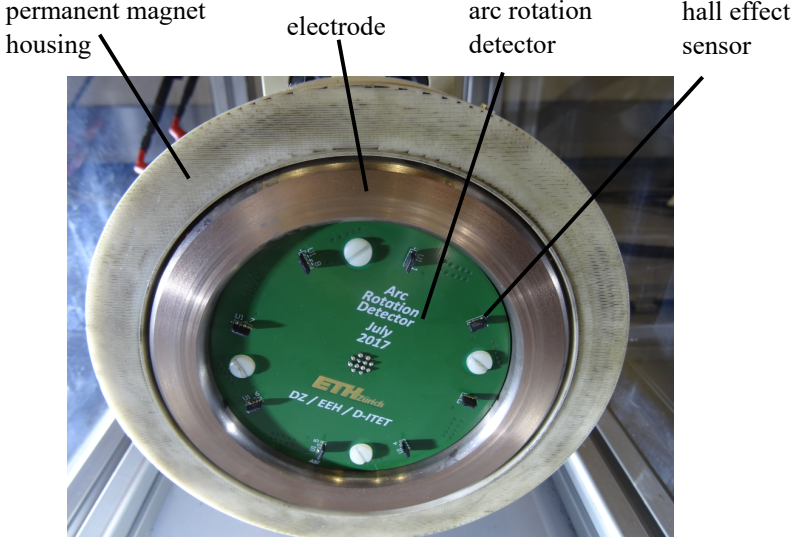

Fig. 6. Frontal view of the fixed contact showing the annular electrode as well as the polycarbonate housing of the permanent magnets and the arc rotation detector in the center.

to generate a transverse magnetic field across a potentially long opening distance can represent major design challenges. To avoid both of these drawbacks, the generation of a strong transverse magnetic field using permanent magnets positioned in the close to the electrodes was evaluated. In magnetostatic finite element method (FEM) simulations, numerous shapes and magnetizations of commercially available permanent magnets were evaluated before arriving at cylindrical neodymium magnets of $40 \mathrm{~mm}$ height and $10 \mathrm{~mm}$ diameter with an axial remanence of $1.26 \mathrm{~T}$ to $1.29 \mathrm{~T}$. An outer ring made up of 36 magnets and an inner ring of 20 magnets were simulated to be either placed around the electrode of the fixed contact (i.e. single-sided) or on either of the two electrodes (dual-sided) as shown schematically in Fig. 7. In these configurations magnetic fields transverse to the arc with a large degree of radial and angular homogeneity resulted. In Fig. 8, the transverse magnetic field $B_{\mathrm{r}}$ simulated at both electrode surfaces as well as the middle of the opening distance are plotted as a function of the opening distance. It is apparent, that the single-sided arrangement is well suited for applications which rely only on short opening distances as the magnetic field at the surface of the moving electrode decays rapidly after only a few tens of millimeters. This fact was verified in preliminary tests which demonstrated that transverse magnetic fields below $50 \mathrm{mT}$ on the electrode of the moving contact lead to sticking of the arc root. Therefore, all tests presented herein were performed using the dual-sided arrangement.

To fix the cylindrical permanent magnets in place while also
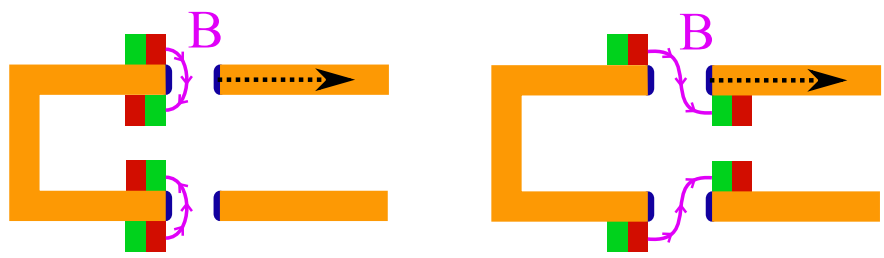

Fig. 7. Schematic view of the single-sided (left) and dual-sided (right) mounting options of the permanent magnets on the fixed and moving contacts. Direction of movement is indicated by dotted arrow. Cylindrical magnets are illustrated as magnetic north (red) and south (green) poles. 


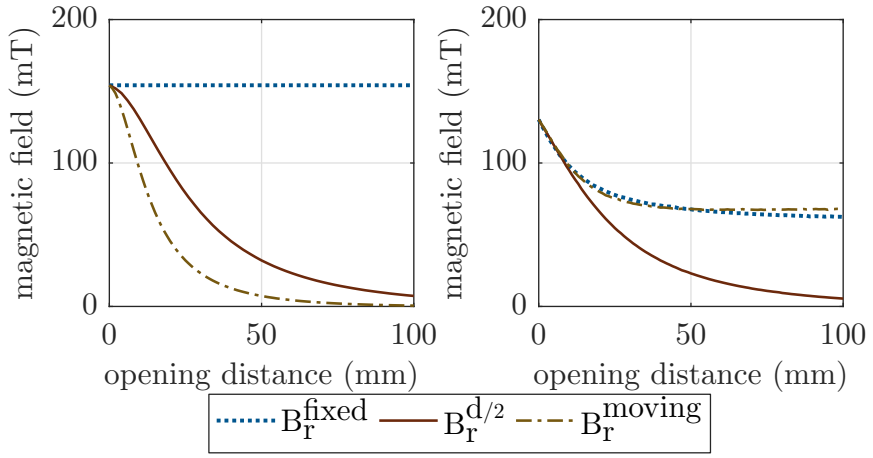

Fig. 8. Simulation of the radial magnetic field at the electrode surfaces of the fixed contact $\left(\mathrm{B}_{\mathrm{r}}^{\mathrm{fixed}}\right)$ and moving contact $\left(\mathrm{B}_{\mathrm{r}}^{\text {moving }}\right)$ as well as halfway between the two contacts $\left(\mathrm{B}_{\mathrm{r}}^{\mathrm{d} / 2}\right)$ for single-sided (left) and dual-sided magnet arrangement (right) for a straight arc with its roots in the middle of the annular electrode rings.

providing dielectric insulation from the electrodes and thermal insulation from the arc, two polycarbonate housings were additively manufactured. The larger of the two can be seen mounted on the fixed contact in Figs. 5 and 6. Through a matrix of holes on both electrodes, the offset of the magnet housing relative to the electrode surface can be adjusted in steps of $1 \mathrm{~mm}$. To be able to measure the rotational speed of the arc during each test, a detector (referred to as arc rotation detector) consisting of eight high frequency hall-effect sensors with integrated constant current sources and amplifiers was built to fit inside either of the electrodes as shown in Fig. 6 . The sensors transduce magnetic fields perpendicular to their surface at $50 \mathrm{mV} \mathrm{mT}^{-1}$. Thus, each sensor was positioned so as to register the passing of the arc as a voltage peak while being largely undisturbed by the background magnetic field caused by the permanent magnets. A total of eight hall-effect sensors were placed at $45^{\circ}$ from each other in order to achieve a high spatial resolution. In post-processing, a peak detection algorithm determined the points in time at which the arc passed by each of the eight sensors, thereby merging all eight voltage signals into one measurement of arc speed of time. Due to the location of the the arc rotation detector, the measured arc speed corresponds to the speed of the arc root on the surface of the electrode within which the detector is mounted.

\section{ViI. Arc Rotation in Air: Speed \& Voltage}

In Fig. 10 two tests at either limit of the opening speed of the testbed are presented with the magnets in the dual-sided arrangement for a constant current of $2.5 \mathrm{kA}$. These tests were performed using FPDCS with the testbed directly connected as load, i.e. without any bus-transfer loop components (c.f. Fig. 2). It is clear from the electrode separation distance measured by the linear motor itself $\left(\mathrm{d}_{\text {meas }}\right)$, that the maximum available pulling force of the linear motor is only enough to reach the desired $4 \mathrm{~m} \mathrm{~s}^{-1}$ towards the end of the fast opening test while the test at $0.1 \mathrm{~m} \mathrm{~s}^{-1}$ exhibits a constant speed over the majority of the arcing time. A delay of $6 \mathrm{~ms}$ before the start of arc rotation was found to be fairly consistent, independent of switching current and opening speed. The deviations in measured arc voltage $\left(\mathrm{u}_{\mathrm{DS}}\right)$ from the fit $\left(\mathrm{u}_{\mathrm{arc}}^{\mathrm{fit}}\right)$ are most likely caused by temporary elongations of the arc column. From high speed images as well as voltage measurements, it can be concluded that the magnitude of these temporary elongations increase with opening distance and decrease with switching current. This property is especially beneficial for switching cases such as bus-transfer, in which an increase in arc voltage accelerates the decrease of the switching current.

The measured arc speeds $\left(\mathrm{m} \mathrm{s}^{-1}\right)$ in the example of Fig. 10 are drawn as a band of possible values: Since the the hall-effect sensors measure in rotations per time $\left(\mathrm{rad} \mathrm{s}^{-1}\right)$, the speed of the lower end of the band corresponds to the arc burning on the inner diameter of the electrode $\left(v_{\text {inner }}\right)$, while the upper end corresponds to the opposite $\left(\mathrm{v}_{\text {outer }}\right)$. The acceleration phase of $10 \mathrm{~ms}$ only seen in the test with slow opening speed can be attributed to two phenomena: Firstly, due to the small opening distance, each rotation of the arc heats up the air between the two electrodes, decreasing the gas density until an equilibrium of heat transfer is reached [35]. Secondly, at arc lengths in the range of a few millimeters, electrode effects play a significant role in determining arc speed. This influence decreases at greater opening distances [32].

The results of a test series of arc speeds recorded at different
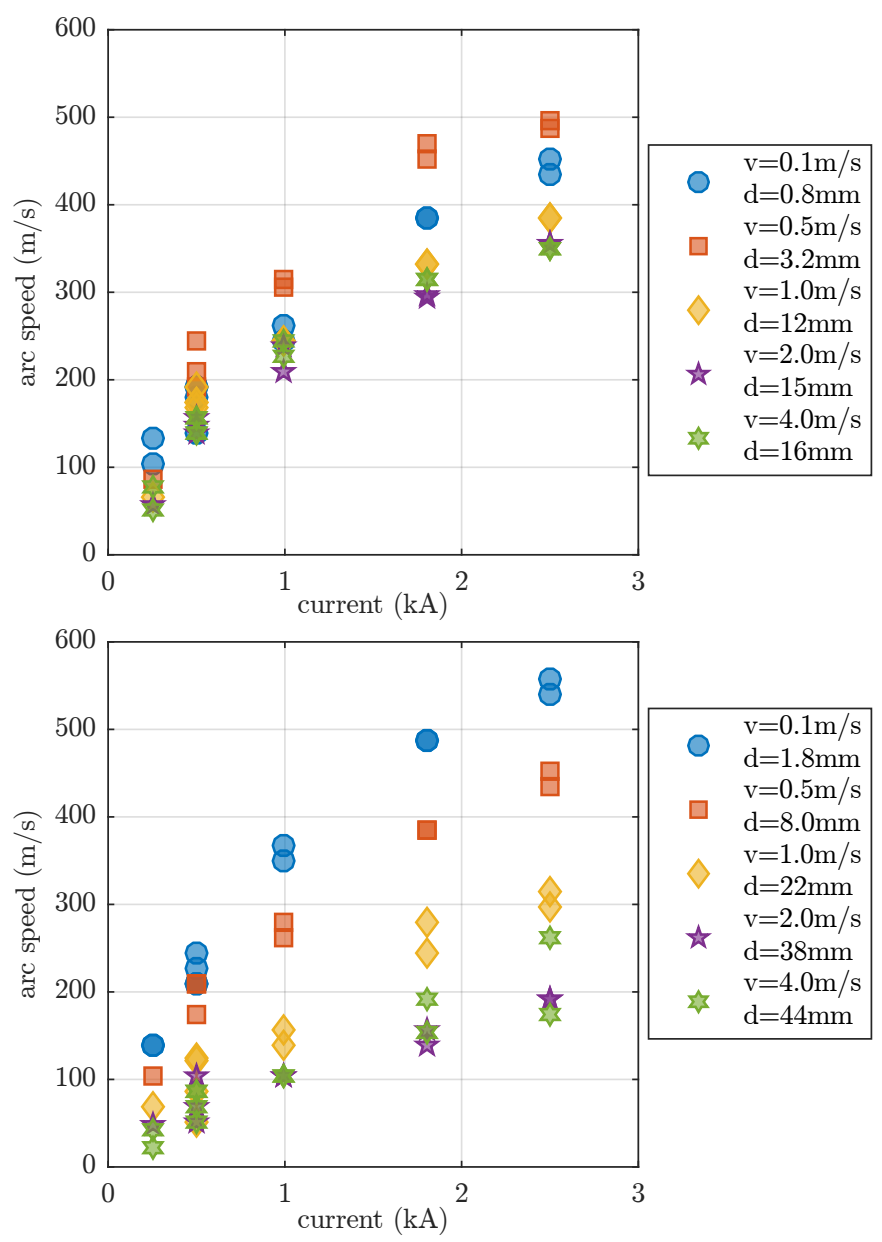

Fig. 9. Arc rotation speeds calculated at the middle of the annular electrodes based on the measured rotation frequencies, averaged at arcing times $10 \mathrm{~ms}$ to $15 \mathrm{~ms}$ (top) and $20 \mathrm{~ms}$ to $25 \mathrm{~ms}$ (bottom) for varying opening speeds $v$.d corresponds to the opening distance in the middle of each arcing time range. 

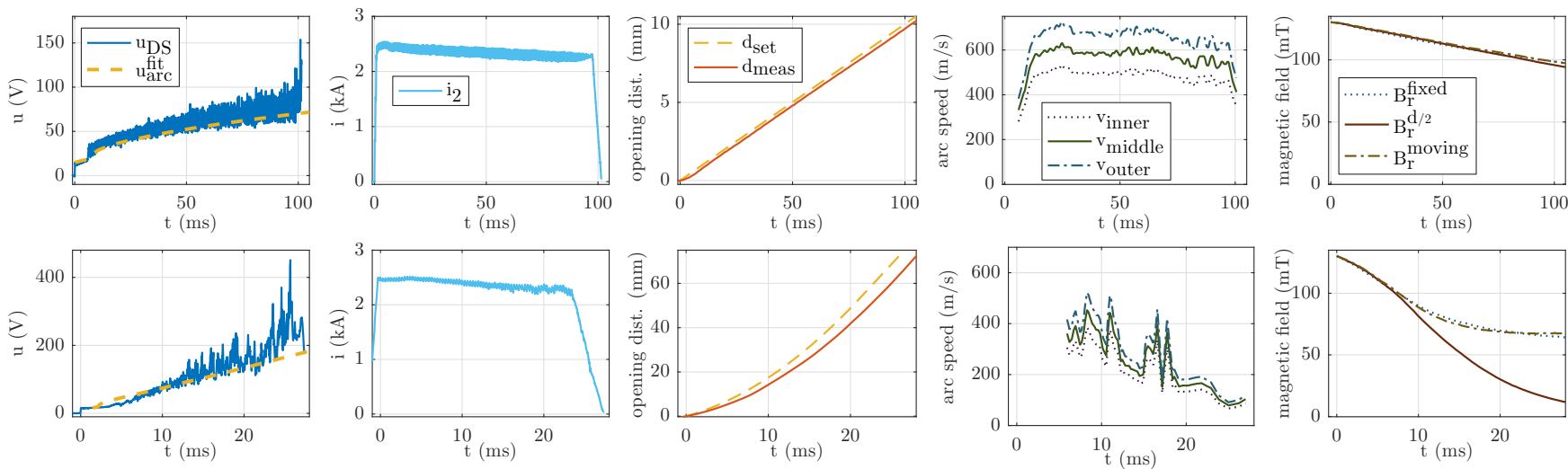

Fig. 10. Comparison of two high-current tests at peak opening speeds of $0.1 \mathrm{~m} \mathrm{~s}^{-1}$ (top) and $4 \mathrm{~ms}^{-1}$ (bottom) and constant currents of $2.5 \mathrm{kA}$ used to evaluate arc speed as a function of current. Legend in upper plots applies to both tests shown.

currents and opening speeds are shown in Fig. 9. The less than linear increase of speed with current can be attributed to the linear increase of Lorentz force with current, which is counteracted by aerodynamic drag growing with the square of the speed. Guile et al. [32] give a proportionality of $v \propto I^{0.4}$, while Szente et al. [33] found $v \propto I^{0.60}$. In the presented data it is also evident that arc speed decreases with opening distance. This is a result of the decreasing magnetic field of the permanent magnets acting on the arc column with increasing opening distance. The cited literature furthermore agrees that changes in the density of the cold gas surrounding the arc can be taken into account as $v \propto \rho_{a}^{-0.5}$, where $\rho_{a}$ is the relative gas density. Therefore, the arc speeds of Fig. 9 gathered in ambient air are approximately five times higher than what would be expected in a typical GIS filled with $\mathrm{SF}_{6}$ pressurized to 5 bar. A less precisely quantifiable effect of the ambient gas is the ability to react chemically with the electrode surface. Oxidation of electrodes has been shown to have a measurable influence on arc speed and on erosion (c.f. [17], [33]). When performing test series using the disconnector testbed, exposing electrodes contaminated with erosion products to air for extended periods led to a substantial increase in arc rotation speed.

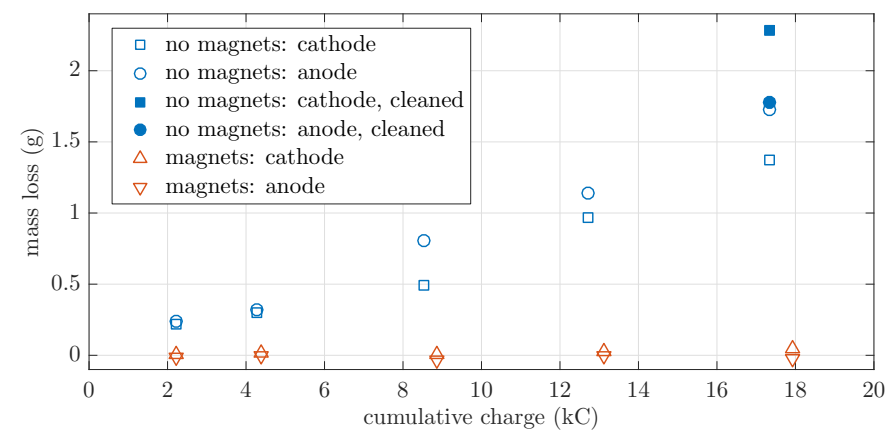

Fig. 11. Measured mass loss during repetitive erosion tests at currents of $1.8 \mathrm{kA}$ to $2.5 \mathrm{kA}$ and arcing times up to $100 \mathrm{~ms}$. Filled symbols represent the mass loss after thoroughly cleaning the surface of the contacts.

\section{ARC Rotation: Electrode Erosion}

One of the major benefits of continuous arc rotation on large electrodes is the distribution of heat transferred into the electrode surfaces and thus the reduction of erosion of the electrode material. To quantify this reduction, the following five series of tests were performed for two sets of identical electrodes in air with magnetic arc driving and an opening speed of $0.1 \mathrm{~m} \mathrm{~s}^{-1}$ :

1) \& 2) $25 \mathrm{x} 1.8 \mathrm{kA}$ for $50 \mathrm{~ms} \approx 2.3 \mathrm{kC}$

3) \& 4) $25 \mathrm{x} 1.8 \mathrm{kA}$ for $100 \mathrm{~ms} \approx 4.5 \mathrm{kC}$

5) $20 \mathrm{x} 2.5 \mathrm{kA}$ for $100 \mathrm{~ms} \approx 5.0 \mathrm{kC}$, c.f. Fig. 10

After each series, the arcing electrodes were removed and weighed individually on a scale with a precision of $50 \mathrm{mg}$. After the final weighing, anode and cathode were thoroughly cleaned in order to remove transferred and redeposited electrode material. The results of these tests are shown in Fig. 11. While the mass lost without magnetic arc driving increases steadily with cumulative charge, there is also a substantial amount of erosion product in the form of spherical drops on the cathode that were only removed after the last test. The total mass lost is $4.1 \mathrm{~g}$, for a cumulative charge of $17.3 \mathrm{kC}$, i.e. an averaged erosion rate of $240 \mu \mathrm{g} \mathrm{C}-1$. After the test series with magnetic arc driving no mass loss was detectable as the results were within the uncertainty of the scale used, i.e. $100 \mathrm{mg}$, resulting in a mass loss of less than $100 \mathrm{mg}$ over $17.9 \mathrm{kC}$ and an erosion rate of less than $5.6 \mu \mathrm{g} \mathrm{C}^{-1}$. Additionally, cracking of the electrode surface and emission of metallic particles was found to be non-existent on these electrodes except for a small region in which the arc preferentially remained stationary for the first few milliseconds of each test.

The difference between erosion is expected to be more accentuated in compressed $\mathrm{SF}_{6}$, as the arc root current densities in the presented tests (executed in air) revealed lower values than known from disconnector application in compressed $\mathrm{SF}_{6}$. Typical concepts to predict erosion balance energy fluxes such as radiation, electrode falls, evaporation and diffusive conduction into the contact bulk. Heat conduction into electrode and contact material increases with the arc root area, shifting the energy flux balance in the direction of lower erosion.

Typical arc root point current densities are about $j=$ 
$10^{8} \mathrm{~A} \mathrm{~m}^{-2}$ in compressed $\mathrm{SF}_{6}$, whereas the densities in the current test have been a third of this value (i.e. increasing the root area by a factor of three). Furthermore, observations using a high speed camera revealed, that only about one third of the tests exhibited a stable, undisturbed arc during the test. One third of the arcs showed slight movement and another third strong, continuous movement during the test in air. Conversely, no such behavior was observed in the tests under compressed $\mathrm{SF}_{6}$, thus larger erosion rates are expected in compressed $\mathrm{SF}_{6}$ conditions due to the increased localized heating of stationary arc roots.

A rough estimation of the impact of the larger arc root diameter is possible by assuming a spherical symmetric heating from the arc root into the material. By further assuming constant heat conduction and capacity of the electrode material (i.e. neglecting temperature dependency), the total heat conduction $P_{\text {cond }}$ into the electrode material scales with the arc root radius $r_{\text {arc }}$ according to

$$
P_{\text {cond }} \propto\left(r_{\text {arc }}+\frac{r_{\text {arc }}^{2}}{\sqrt{\pi c_{\mathrm{p}} \lambda t}}\right)
$$

with the arcing time $t$, the specific heat $c_{\mathrm{p}}$, and the thermal conductivity $\lambda$. Hence, the arc root enters linearly for long arcing time (stationary temperature profile) and quadratically at the beginning (transient temperature profile). Hence, the erosion rates measured in atmospheric air (c.f. Fig. 11) are expected to a factor of $1.5 \ldots 3$ higher under the condition of compressed $\mathrm{SF}_{6}$ (with three time larger arc root current densities).

\section{Arc Rotation: Bus-Transfer}

In addition to the erosion tests, a number of bus-transfer tests were performed at opening speeds of $0.1 \mathrm{~m} \mathrm{~s}^{-1}$ using the same setup and evaluation methods as for the fast-acting disconnector. The control tests without magnetic arc driving revealed substantial differences in reignition behavior to the previously studied GIS disconnectors of the same opening speed operating in compressed $\mathrm{SF}_{6}$. In some cases, the conditions for immediate reignition at current zero appeared to vanish faster, leading to extinctions at recovery voltages up to ten times higher than the observed arc voltage. This difference is likely due to the 27 times lower density of ambient air compared to pressurized $\mathrm{SF}_{6}$, which increases the mobility of the arc column and accelerates the diffusion of hot gas into the area surrounding the arc. Furthermore, delayed reignitions up to $500 \mu$ s after current zero at voltages in the range of $400 \mathrm{~V}$ to $600 \mathrm{~V}$ were recorded, most likely caused by the lower dielectric strength of hot ambient air compared to hot $\mathrm{SF}_{6}$ and the superior cooling properties of the latter. Nevertheless, numerous immediate reignitions, charging reignitions and delayed reignitions were recorded at levels comparable to prior GIS tests.

With the permanent magnets mounted for magnetic arc driving, the bus-transfer results are split into two categories. For the initial few milliseconds in which the arc remained stationary at the point of inception, no change compared to the tests without magnets is apparent, i.e. reignitions occur in the same manner at the same voltages. However, once arc rotation started, no more immediate or charging reignitions were registered. It can be assumed that the rapid movement of the arc leads to an efficient mixing of hot and ambient gas at current zero, thus rapidly eliminating the conditions favoring reignition. The same has been found for delayed reignitions in all but one case, even at voltages that were guaranteed to reignite the electrode gap without magnets. The one exception was recorded when a current zero occurred immediately after the start of arc rotation before a full rotation was completed. The switching arc reignited at the point of original arc inception instead of the position at current zero, most likely due to the localized heating occurring for $6 \mathrm{~ms}$ preceding the start of the arc movement. This change in reignition behavior represents a fundamental improvement of the switching capabilities for $\mathrm{HVdc}$ applications. Without reignitions at current zero (within reasonable voltage ranges), the time to first current zero automatically becomes the total switching time.

\section{Conclusions}

It has been shown with a combination of simulations and large test series that readily available HVac MTS disconnectors offer advantages over traditional slow-moving GIS disconnectors for MTdc switching cases such as bus-transfer. The fast increase in arc voltage over time leads to a short time to first current zero, even for extremely large sections of GIS at high currents, while also providing improved resilience against recovery voltages caused by current harmonics. Furthermore, an arrangement of widely available permanent magnets, which requires only minimal changes to proven disconnector designs, has been shown to be able to drive switching arcs at up to $600 \mathrm{~m} \mathrm{~s}^{-1}$ in air. The efficacy of magnetic arc driving has been demonstrated for reducing electrode erosion while at the same time increasing switching performance.

Both of these technologies unlock further possibilities in terms of switching cases and applications of disconnectors in future MTdc substations and grids.

\section{ACKNOWLEDGMENTS}

The authors would like to express their gratitude to Alexander Heller, Stefan Franz and Verena Häberle for their contributions in advancing the magnetic arc driving project.

\section{REFERENCES}

[1] Cigré Working Group D1.03, "Gas Insulated Systems for HVDC: DC Stress at DC and AC Systems," Cigré Brochure, no. 506, p. 79, 2012.

[2] T. Shimato, T. Hashimoto, and M. Sampei, "The Kii Channel HVDC Link in Japan," Cigré Session, no. 14-106-2002, 2002.

[3] R. Alvinsson, E. Borg, A. Hjortsberg, T. Hoglund, and S. Hornfeldt, "GIS for HVDC Converter Stations," Cigré Session, no. 14-02-1986, 1986.

[4] S. Rodrigues, C. Restrepo, E. Kontos, R. Teixeira Pinto, and P. Bauer, "Trends of offshore wind projects," Renewable and Sustainable Energy Reviews, vol. 49, pp. 1114-1135, 2015.

[5] C. M. Franck, "HVDC Circuit Breakers: A Review Identifying Future Research Needs," IEEE Transactions on Power Delivery, vol. 26, no. 2, pp. 998-1007, apr 2011.

[6] M. Callavik, A. Blomberg, J. Häfner, and B. Jacobson, "Break through! ABB's hybrid HVDC breaker," ABB Review, no. 2, pp. 7-13, 2013. 
[7] C. Davidson, R. Whitehouse, C. Barker, J.-P. Dupraz, and W. Grieshaber, "A new ultra-fast HVDC Circuit breaker for meshed DC networks," in 11th IET International Conference on AC and DC Power Transmission. Institution of Engineering and Technology, 2015.

[8] G. Tang, Z. He, H. Pang, X. Huang, and X.-p. Zhang, "Basic topology and key devices of the five-terminal DC grid," CSEE Journal of Power and Energy Systems, vol. 1, no. 2, pp. 22-35, jun 2015.

[9] G. Tang, "High Power Conversion Technology for High Voltage DC Transmission Application," in IEEE Energy Conversion Congress \& Expo (ECCE) 2016, 2016, pp. 1-45.

[10] Cigré Working Group J W G A3/B4.34, "Technical Requirements and Specifications of State-of-the-Art HVDC Switching Equipment," JWG A3/B4,34, Tech. Rep. April, 2017.

[11] Cigré Working Group J W G 13/14.08, "The current commutation function of HVDC switching devices," Cigré Electra, no. ELT-124-2, 1989.

[12] P. Vinson, A. Girodet, A. Hanouna, and S. Poullain, "Investigations of load transfer capabilities of GIS disconnectors for DC grids," in 3rd International Conference on Electric Power Equipment - Switching Technology (ICEPE-ST 2015), 2015.

[13] A. Ritter and C. M. Franck, "Prediction of Bus-Transfer Switching in Future HVDC Substations," IEEE Transactions on Power Delivery, vol. 8977 , no. c, pp. 1-1, 2017.

[14] A. Ritter, U. Straumann, and C. M. Franck, "Novel Method for Predicting Limit Performance of Bus-Transfer Switching by Disconnectors," IEEE Transactions on Power Delivery, vol. 32, no. 5, 2017.

[15] Cigré Working Group A3.06, "Final Report of the 2004 - 2007 International Enquiry on Reliability of High Voltage Equipment - Part 5 Gas Insulated Switchgear (GIS)," Cigré Brochure, vol. 513, no. October 2012, 2012.

[16] J. J. Shea, "High Current AC Break Arc Contact Erosion," in 2008 Proceedings of the 54th IEEE Holm Conference on Electrical Contacts, vol. 00, no. c. IEEE, 2008, pp. xxii-xlvi.

[17] A. Guile and B. Jüttner, "Basic Erosion Processes of Oxidized and Clean Metal Cathodes by Electric Arcs," IEEE Transactions on Plasma Science, vol. 8, no. 3, pp. 259-269, sep 1980.

[18] A. Guile, "Electric arcs: their electrode processes and engineering applications," IEE Proceedings A Physical Science, Measurement and Instrumentation, Management and Education, Reviews, vol. 131, no. 7, p. 450, 1984.

[19] N. Fujimoto, "Conduction Currents in Gas-Insulated Switchgear For Low Level DC Stress," in Gaseous Dielectrics V. Elsevier, 1987, pp. 513-519.

[20] X. Jun and I. D. Chalmers, "The influence of surface charge upon flash-over of particle-contaminated insulators in under impulse-voltage conditions," Journal of Physics D: Applied Physics, vol. 30, no. 7, pp. 1055-1063, apr 1997.

[21] Cigré Working Group D1.03, "Gas Insulated Systems for HVDC: DC Stress at DC and AC Systems," Cigré Brochure, no. 506, p. 79, 2012.

[22] M. Shikata, K. Yamaji, M. Hatano, R. Shinohara, F. Endo, and T. Yamagiwa, "Insulation Characteristics of DC500kV GIS," in Gaseous Dielectrics VIII. Boston, MA: Springer US, 1998, pp. 573-579.

[23] M. Holmberg and S. Gubanski, "Motion of metallic particles in gas insulated systems," IEEE Electrical Insulation Magazine, vol. 14, no. 4, pp. 5-14, jul 1998.

[24] Y. Negara, K. Yaji, J. Suehiro, N. Hayashi, and M. Hara, "DC corona discharge from floating particle in low pressure SF/sub 6/," IEEE Transactions on Dielectrics and Electrical Insulation, vol. 13, no. 6, pp. 1208-1216, dec 2006

[25] M. M. Walter, Switching arcs in passive resonance HVDC circuit breakers. Diss. ETH-Zürich, 2013.

[26] A. Ritter, L. S. J. Bort, and C. M. Franck, "Five years of pulsed current testing for HVDC switchgear," in 2016 IEEE International Conference on High Voltage Engineering and Application (ICHVE). IEEE, sep 2016, pp. 1-4.

[27] L. Dalessandro, N. Karrer, M. Ciappa, A. Castellazzi, and W. Fichtner, "Online and offline isolated current monitoring of parallel switched highvoltage multi-chip IGBT modules," in 2008 IEEE Power Electronics Specialists Conference. IEEE, jun 2008, pp. 2600-2606.

[28] A. Ritter, U. Straumann, U. Riechert, and C. M. Franck, "Analysis Method and Implications of Utilizing a Full Bus-Transfer Setup for Testing Disconnectors," in 2015 Cigré SC A3\&B3 Joint Colloquium, 2015.

[29] J. S. Pearson, B. F. Hampton, M. D. Judd, D. Templeton, and I. M. Welch, Gas Insulated Substations, H. Koch, Ed. Chichester, United Kingdom: John Wiley \& Sons Ltd, jul 2014, no. 5.
[30] C. Siegenthaler, M. Keller, M. Boesch, U. Kruesi, and R. Kallweit, "Electrical high voltage circuit breaker and method for opening same," European Patent Office Patent EP2 544 203, 2013.

[31] S. Franz, "Optimizing HVDC Disconnectors for Bus-Transfer Switching," Master Thesis, ETH Zürich, 2016.

[32] A. Guile, V. Adams, W. Lord, and K. Naylor, "High-current arcs in transverse magnetic fields in air at atmospheric pressure," Proceedings of the Institution of Electrical Engineers, vol. 116, no. 4, p. 645, 1969.

[33] R. N. Szente, R. J. Munz, and M. G. Drouet, "Arc velocity and cathode erosion rate in a magnetically driven arc burning in nitrogen," Journal of Physics D: Applied Physics, vol. 21, no. 6, pp. 909-913, jun 1988.

[34] S. Yanabu, S. Nishiwaki, H. Mizoguchi, N. Shimokawara, and Y. Murayama, "High Current Interruption by SF6 Disconnecting Switches in Gas Insulated Switchgear," IEEE Transactions on Power Apparatus and Systems, vol. PAS-101, no. 5, pp. 1105-1114, may 1982.

[35] J. Kopainsky and E. Schade, "Rotating high current arc," Applied Physics, vol. 20, no. 2, pp. 147-153, oct 1979.

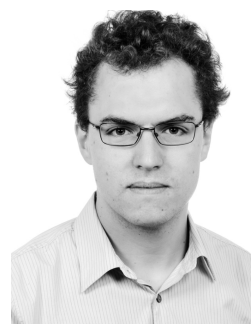

Andreas Ritter (M09) was born in Basel, Switzerland, in 1986. He received the M.Sc. degree in electrical engineering with distinction from ETH Zurich, Zurich, Switzerland, in 2012. He is currently a scientific assistant at the High Voltage Laboratory at ETH Zurich, researching disconnector and earthing switch operation in future multiterminal $\mathrm{HVdc}$ grids.

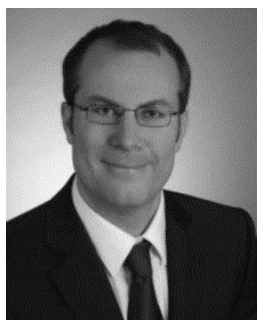

Ueli Straumann (M10SM12) was born in Aarau, Switzerland, in 1975. He received the Ph.D. degree in electrical engineering from ETH Zurich, Zurich, Switzerland, in 2007. Since then, he has been a Lecturer of High Voltage Engineering, ETH Zurich. After being Senior Assistant at the High Voltage Laboratory of ETH Zurich between 2007 and 2012, he became Senior and Principal Engineer at the GIS R\&D of ABB Switzerland Ltd.

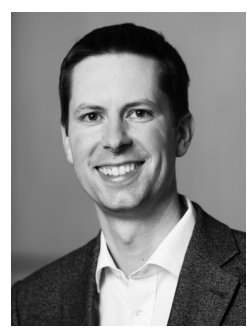

Christian M. Franck (M04 - SM11) received a diploma in physics from the University of Kiel, Germany in 1999 and the Ph.D. degree in physics from the University of Greifswald, Germany in 2003. He was with the Swiss corporate research center of ABB during 2003-2009 as a Scientist and Group Leader for gas circuit breakers and high voltage systems. Currently, he is Associate Professor for High Voltage Technology at the Swiss Federal Institute of Technology (ETH), Zurich, Switzerland. His main research interests are in the area of gaseous insulation systems and equipment for $\mathrm{HVdc}$. 